\title{
Precalibration Versus 2D-3D Registration for 3D Guide Wire Display in Endovascular Interventions
}

\author{
Shirley A.M. Baert, Graeme P. Penney, Theo van Walsum, and Wiro J. Niessen \\ Image Sciences Institute, University Medical Center Utrecht \\ Rm E 01.335, P.O.Box 85500, 3508 GA Utrecht, The Netherlands \\ \{shirley, graeme, theo, wiro\}@isi.uu.nl
}

\begin{abstract}
During endovascular interventions, the radiologist relies on $2 \mathrm{D}$ projection images to advance the guide wire, while often a preoperative 3D image of the vasculature is available. To take full advantage of the 3D information, two different methods are proposed and compared for $3 \mathrm{D}$ guide wire reconstruction and visualization in the $3 \mathrm{D}$ vasculature. Upon tracking the guide wire in biplane fluoroscopy images, the first approach utilizes a precalibrated C-arm system to determine the guide wire position in the $3 \mathrm{D}$ coordinate system of the vasculature. In the second method the relation between the projection images and the $3 \mathrm{D}$ vascular data is determined using intensity based 2D-3D registration. Based on a study on an anthropomorphic phantom, it is shown that the calibration method is highly accurate, but that in case of imperfect geometry knowledge results rapidly degrade. For 2D-3D registration, similar accurate results were obtained with a $97.8(84.2) \%$ success rate if the registration starting position was within 4 (8) degrees rotation and 5 (10) mm translation of the reference position. The latter method has also been used to succesfully reconstruct a guide wire in a patient dataset.
\end{abstract}

\section{Introduction}

During endovascular interventions, it is important for the radiologist to accurately know the 3D position of the guide wire at any time during the procedure. Currently, 2D fluoroscopic projection images are used for navigation. Therefore, a mental reconstruction of the position and orientation of the guide wire in $3 \mathrm{D}$ has to be performed, which can be a difficult task. With the introduction of motorized calibrated X-ray angiography, a 3D reconstruction of the vasculature can be obtained immediately prior to the intervention. Visualizing the guide wire in the $3 \mathrm{D}$ vasculature could potentially be used as an additional navigation tool for the radiologist. One method is to track the guide wire in the biplanar fluoroscopic images to reconstruct its position in $3 \mathrm{D}$.

In order to produce a $3 \mathrm{D}$ reconstruction of the guide wire and relate it to the $3 \mathrm{D}$ coordinate system of the $3 \mathrm{D}$ vascular data, accurate knowledge of the C-arm geometry is required. To this end, a wide range of calibration procedures have been proposed. In this paper two approaches are proposed and compared. In the 
first approach, the fact that the interventions are carried out on a fixed system with reproducible geometry is exploited. The system geometry is estimated in a precalibration step [1] that only has to be carried out once. The advantage of this approach is that it does not hamper intraoperative logistics or image quality, as no changes have to be made to the intraoperative situation. A disadvantage is that to maintain the relation between the $3 \mathrm{D}$ vascular data and the projection images, the patient should be stabilized or tracked during the intervention. In the second approach, anatomical information that is contained in the images is used to relate the $3 \mathrm{D}$ vascular data to projection images. There are various methods to achieve this, and in this paper intensity-based 2D-3D registration is considered.

\section{Calibration Methods}

\subsection{Precalibration}

For the precalibration, the calibration method of the 3D rotational angiography facility on the Philips Integris BV5000 is used (see 1]). In this calibration step the geometry of the system and the image distortions are determined for a reproducible 3D rotational run over 180 degrees of the $\mathrm{C}$-arm. Two types of distortion are present, pincushion distortion caused by the curved input screen on the image intensifier and s-shaped distortion caused by interactions between the earth's magnetic field and electrons in the image intensifier. The distortion is measured using a Cartesian-grid phantom with equally spaced grid-points and is modelled using bivariate polynomials. Distortion correction is performed to subpixel accuracy. The projection geometry is measured for each projection angle using the same grids and a calibration phantom, since the isocenter position is not constant due to mechanical bending of the $\mathrm{C}$-arm.

\subsection{Image-Based Calibration}

For the image-based calibration, a DSA sequence that is routinely made in cerebral interventions for both the frontal and lateral C-arm, is registered with the priorly obtained 3D volume of the vasculature. We have used an intensity based 2D-3D registration algorithm [2]3 to register the pre-operative 3DRA data to the interventional DSA images. The algorithm produces digitally reconstructed radiographs (DRR's) which are compared to the DSA image using a similarity measure (gradient difference). The geometry information is comprised of two sets of parameters, intrinsic parameters which define the projection geometry of the fluoroscopy set and extrinsic parameters ( 3 rotational and 3 translational) which define the pose and position of the $3 \mathrm{D}$ volume with respect to the fluoroscopy set. The algorithm assumes that the intrinsic parameters are known, and alters the extrinsic parameters, using a gradient descent type search strategy, in order to optimize the similarity measure. A circular region of interest is defined in the DSA images in order to limit the registration to a particular region of the image 
and to speed up the algorithm. Since in general there is also no knowledge of the relative position between the C-arms, the procedure is carried out for both biplane images separately, which means that a monoplane $2 \mathrm{D}-3 \mathrm{D}$ registration is performed twice and independently. A small modification has been made to the algorithm as presented in 23. in order to improve its capture range: a global search varying only in plane translations (up to $20 \mathrm{~mm}$ in steps of $2 \mathrm{~mm}$ ) and rotations (up to 20 degrees in steps of 4 degrees) is carried out prior to the full six degree of freedom optimization. DRR's produced at different stages in the registration process and a target DSA image are shown in Figure 1.
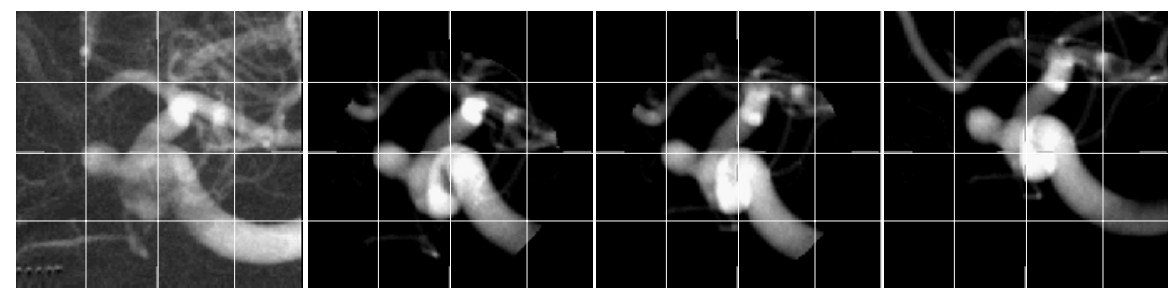

Fig. 1. Target DSA image (left) and three DRR's produced during the registration process; (from left to right) a DRR produced at the final registration position, after the initial in-plane registration, and at the starting position, respectively.

\section{Experiments}

After establishing the geometry information with either of the proposed methods, the 3D reconstruction of the guide wire is performed in three steps. Upon (i) tracking the guide wire in 2D biplane fluoroscopic images, the estimated geometry is used (ii) to determine corresponding points in both projections for guide wire reconstruction, and to (iii) show the guide wire together with the preoperatively acquired $3 \mathrm{D}$ vasculature. Information on the tracking method and 3D reconstruction method can be found in [45]. An accuracy study on a phantom and a feasibility study in a clinical case have been carried out.

\subsection{Phantom Experiment}

For all the experiments 3D runs over 180 degrees containing 100 projection images were made of an intracranial anthropomorphic vascular phantom using the rotational angiography facility of a Philips Integris BV5000 C-arm imaging system. Initially the phantom is filled with contrast to obtain a pre-operative $3 \mathrm{D}$ vasculature image $\left(256 \times 256 \times 256\right.$ voxels of size $\left.0.521 \times 0.521 \times 0.521 \mathrm{~mm}^{3}\right)$, see Figure 2. After removing the contrast, a guide wire was advanced in 24 approximately equally spaced steps. After each advance a $3 \mathrm{D}$ reconstruction 

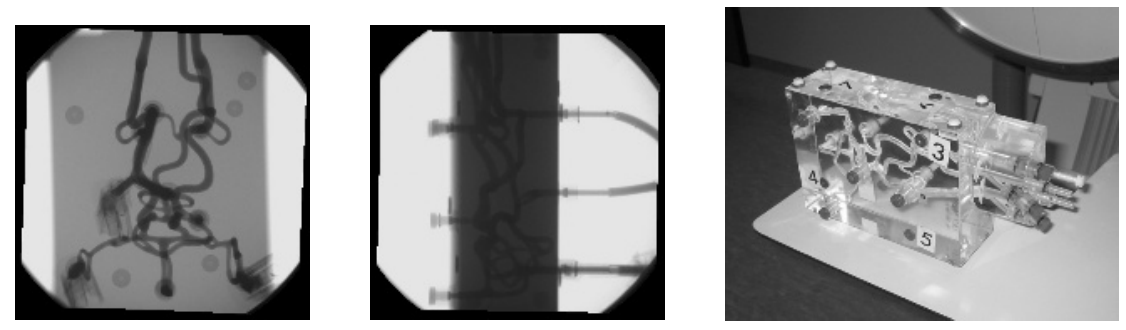

Fig. 2. Left and middle: two fluoroscopic images out of a 3D run showing the anthropomorphic vascular phantom (right) in frontal and lateral position.

was made. The extracted guide wire in these sequences servers as ground truth for the experiments.

From each 3DRA acquisition 19 of the 2D fluoroscopy images were extracted at 10 degree intervals between 0 and 180 degrees. Sets of fluoroscopy images, acquired at the same angle but at different time intervals, were used to represent a dynamic fluoroscopy sequence (each image has $512 \times 512$ pixels of size $\left.0.527 \times 0.527 \mathrm{~mm}^{2}\right)$. All images were corrected for distortion.

Experiments were carried out to determine the accuracy of the precalibartion based guide wire reconstruction as a function of the angle between the biplane images. Additionally, the influence of the accuracy of the precalibration was investigated.

Similar experiments were carried out for the image-based calibration. To estimate the capture range of the method, different starting positions were generated by altering the position of the $3 \mathrm{D}$ dataset from the precalibrated position using four different perturbations, consisting of $\pm 2^{\circ}, \pm 4^{\circ}, \pm 8^{\circ}, \pm 12^{\circ}$ rotation and $\pm 2.5 \mathrm{~mm}, \pm 5 \mathrm{~mm}, \pm 10 \mathrm{~mm}, \pm 15 \mathrm{~mm}$ translation, respectively. For each of these four perturbations, ten starting points were picked by randomly altering all of the six extrinsic parameters by either + or - the perturbation. In the experiments using image-based calibration, we used the distortion estimation from the precalibration method, to correct the images. If precalibration is only available in a number of $\mathrm{C}$-arm positions, interpolation can be used, or other methods that have been proposed in the literature [6] can be applied.

\subsection{Patient Experiment}

Prior to a neuro-intervention a 3DRA image of the cerebral vasculature of the patient was made. At the beginning of the intervention, biplane DSA images were acquired for both $\mathrm{C}$-arm positions, which were registered to the 3DRA volume (voxel size $0.385 \times 0.385 \times 0.385 \mathrm{~mm}^{3}$ ) of the vasculature of the patient. During the intervention biplane fluoroscopy images were acquired (pixel size frontal images $0.278 \times 0.278 \mathrm{~mm}^{2}$ and pixel size lateral images $0.423 \times 0.423 \mathrm{~mm}^{2}$ ) while advancing the guide wire in the vasculature, on which the $2 \mathrm{D}$ guide wire tracking method was performed. An estimate of the source image distance, rotation and 
angulation of the $\mathrm{C}$-arm system was obtained for both images from the C-arm system display. These values were used as a starting estimate for the registration algorithm.

\section{Results}

An example of the 3D reconstruction of the guide wire in the phantom images using precalibration is shown in Figure 3 Image-based calibration gives qualitatively similar results.
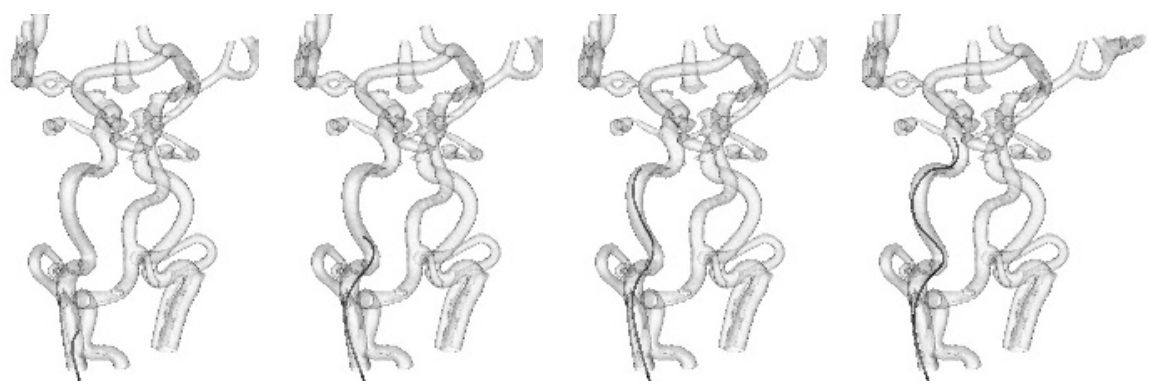

Fig. 3. Four frames (frame 2, 10, 16 and 24) out of a sequence of 24 frames, which gives an impression of the reconstruction results. The reconstructed guide wire position is within the vasculature in all cases.

\subsection{Precalibration}

The left graph in Figure 4 shows the mean distance between the estimated guide wire position and the reference position as a function of the angle between the biplane images and the errors in the precalibrated geometry. Using the precalibrated geometry, the mean distance is smaller than $0.5 \mathrm{~mm}$ and increases if the angle between the biplane images becomes very small $(<30$ degrees $)$ or very large ( $>150$ degrees). If an inaccuracy is introduced in the angulation, the error increases significantly. Table 1 presents the mean distance for all different combinations of inaccuracies in rotation and angulation, averaged over all experiments in the angular range from 30 to 150 degrees.

\subsection{Image-Based Calibration}

The right part of Figure 4 shows the mean errors in 3D guide wire reconstruction that are obtained with 2D-3D registration as image-based calibration method. Registration of the 2D DSA image with the 3DRA volume sometimes fails, especially when the starting position is located further away from the reference standard. If the distance between the estimated position of the focal spot after 

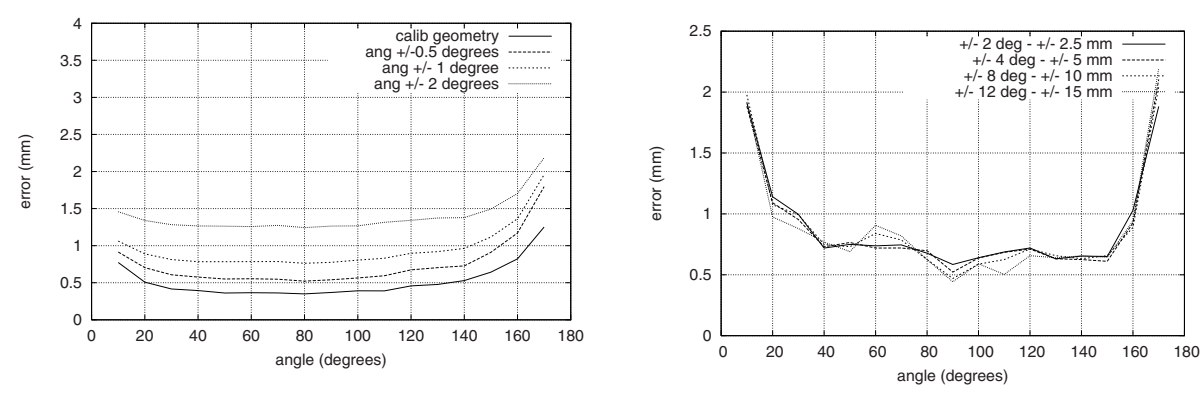

Fig. 4. Mean distance between the automatic reconstruction method and the manual 3DRA position segmentation for different angles between the biplane images (10-170 degrees) using precalibration (left) and image-based calibration (right).

Table 1. Distance in millimeters between the automatically and the manually obtained guide wire position using precalibration information. The mean distance and tip distance (in brackets) is taken over the angles from 30 to 150 degrees. The error is given if the calibrated geometry is used, and if errors $(0.5,1$ and 2 degrees for rotation and angulation) in the accuracy of the geometry are introduced.

\begin{tabular}{lcccc}
\hline \hline & Calibr. geom. & $\operatorname{rot} 0.5^{\circ}$ & $\operatorname{rot} 1^{\circ}$ & $\operatorname{rot} 2^{\circ}$ \\
\hline \hline Calibr. geom. & $0.42[0.65]$ & $0.60[0.93]$ & $0.79[1.23] 1.37[1.88]$ \\
\hline ang $0.5^{\circ}$ & $0.62[0.94]$ & $0.75[1.10]$ & $0.99[1.37]$ & $1.57[2.03]$ \\
\hline ang $1^{\circ}$ & $0.85[1.30]$ & $0.96[1.40]$ & $1.17[1.64]$ & $1.74[2.29]$ \\
\hline ang $2^{\circ}$ & $1.31[1.92]$ & $1.43[2.11]$ & $1.62[2.35]$ & $2.12[2.93]$ \\
\hline \hline
\end{tabular}

registration and the focal spot position for the reference standard is larger than 3 centimeters, we define the registration to be unsuccessful. The unsuccessful registrations were not included in the calculation of the mean and tip distance of the $3 \mathrm{D}$ guide wire reconstruction. It can be observed that for small and large angles, the errors increase. Furthermore it can be observed that approximately the same minimum is found for all the successful registration regardless of the starting position.

Table 2 summarizes the mean and tip distances for the image-based calibration and lists the registration success rates. Values are averaged over 30 to 150 degrees angles, since the results are almost constant over this range. It can be observed from Table 2 that the geometry can be estimated using 2D-3D registration as accurately as by precalibration if a reasonable initialization is available. The success rate of the registrations falls off rapidly once the starting position exceeds $8^{\circ}$ rotation and $10 \mathrm{~mm}$ translation from the reference standard.

Figure 5 shows the results if $2 \mathrm{D}-3 \mathrm{D}$ intensity based registration is used to determine the geometry information and the correspondence between the $2 \mathrm{D}$ 
Table 2. Distance in millimeters between the automatically and the manually obtained guide wire position using image-based calibration. The mean distance and tip distance is taken over the angles from 30 to 150 degrees.

\begin{tabular}{lccc}
\hline \hline Starting position & Mean dist. & Tip dist. & Success rate \\
\hline \hline $\pm 2^{\circ}- \pm 2.5 \mathrm{~mm}$ & 0.42 & 0.71 & $100 \%$ \\
\hline $\pm 4^{\circ}- \pm 5 \mathrm{~mm}$ & 0.39 & 0.69 & $97.9 \%$ \\
\hline $\pm 8^{\circ}- \pm 10 \mathrm{~mm}$ & 0.40 & 0.70 & $84.2 \%$ \\
\hline $\pm 12^{\circ}- \pm 15 \mathrm{~mm}$ & 0.42 & 0.67 & $55.8 \%$ \\
\hline \hline
\end{tabular}

fluoroscopy images and the 3D volume in a clinical case. Owing to patient and table movement, which often occurs in our current clinical setup, only the imagebased calibration procedure could be performed. It can be observed that the estimated position is within the vasculature. In the sixth image (lower right) a visualization is shown of a $3 \mathrm{D}$ reconstruction where the guide wire was manually outlined in the biplane projection images. Quantitative validation is not possible as no 3DRA image is acquired with the guide wire in the vasculature.

\section{Discussion}

$3 \mathrm{D}$ information on the position of the guide wire with respect to the vasculature has the potential to improve the speed, accuracy and safety of endovascular interventions. Therefore, two methods for relating the projection images visualizing the guide wire to the preoperative obtained vasculature are described. The method based on precalibration requires that once the $3 \mathrm{D}$ vascular image is made, the patient should not move, or his motion should be tracked. The imagebased registration method can be used if accurate precalibration information is not available or has become invalid due to patient and/or table movement.

Based on phantom experiments, it was concluded that for projection images with a relative angle in a large range (between 30 and 150 degrees) both methods achieved a mean distance error of approximately $0.4 \mathrm{~mm}$ and a tip distance of approximately $0.7 \mathrm{~mm}$ distance. For the precalibration method, the errors significantly increased if small inaccuracies in rotation and angulation were introduced, stressing the need for accurate calibration. For image-based calibration, the success rate depends on the initial starting positions. Byrne et al [7] have shown in a clinical setting for 10 patients, that patient movement between the $3 \mathrm{D}$ acquisition and the $2 \mathrm{D}$ intervention was within $\pm 4^{\circ}$ rotation for 7 patients and within $\pm 8^{\circ}$ rotation for the other 3 patients. In our experiments, for starting positions within $4^{\circ}$ rotation and $5 \mathrm{~mm}$ translation in all directions, a success rate of $97.8 \%$ was obtained and for starting positions within $8^{\circ}$ rotation and $10 \mathrm{~mm}$ translation, a success rate of greater than $84 \%$ could be obtained, which implies that image-based calibration could potentially be used in clinical practice. 

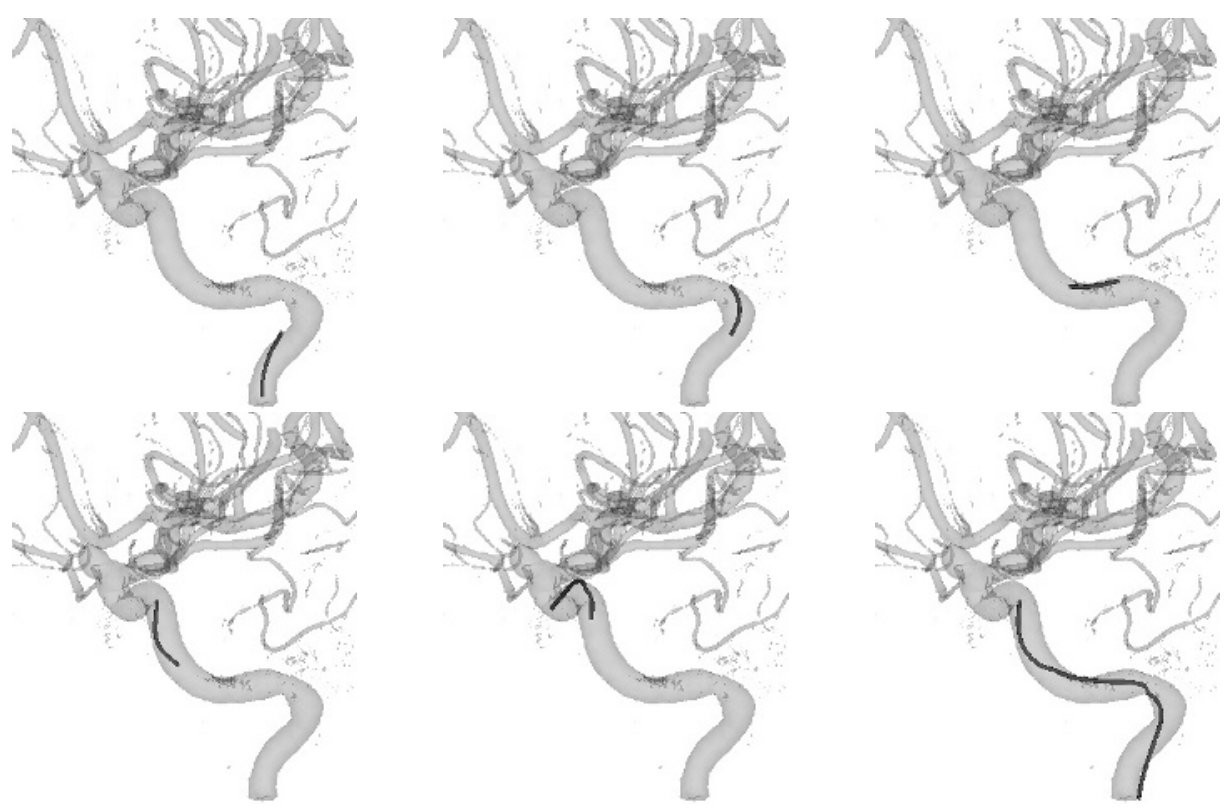

Fig. 5. Five non-subsequent frames of a patient image sequence. Only the tip is shown since in the 2D guide wire tracking only the proximal part of the guide wire is localized. The sixth image (lower right) is a $3 \mathrm{D}$ reconstruction from a manually outlined guide wire in the $2 \mathrm{D}$ projection images.

\section{References}

1. Koppe, R., Klotz, E., de Beek, J.O., Aerts, H.: 3D vessel reconstruction based on rotational angiography. In Lemke, H., Inamura, K., Jaffe, C., Vannier, M., eds.: Proceedings of CAR'95, Springer-Verlag, Berlin (1995) 101-107

2. Hipwell, J., Penney, G., Cox, T., Byrne, J., Hawkes, D.: 2D-3D intensity based registration of DSA and MRA - a comparison of similarity measures. In Dohi, T., Kikinis, R., eds.: Proceedings of MICCAI 2002, part II. Volume 2489 of Lecture Notes in Computer Science., Springer Verlag, Berlin (2002) 501-508

3. Penney, G., Batchelor, P., Hill, D., Hawkes, D., Weese, J.: Validation of a two- or three-dimensional registration algorithm for aligning preoperative CT images and intraoperative fluoroscopy images. Medical Physics 28 (2001) 1024-1032

4. Baert, S., Viergever, M., Niessen, W.: Guide wire tracking during endovascular interventions. IEEE Transactions on Medical Imaging 22 (2003) 965-972

5. Baert, S., van de Kraats, E., van Walsum, T., Viergever, M., Niessen, W.: 3D guide wire reconstruction from biplane image sequences for integrated display in 3D vasculature. IEEE Transactions on Medical Imaging 22 (2003) 1252-1258

6. Cañero, C., Vilariño, F., Mauri, J., Radeva, P.: Predictive (un)distortion model and 3 -d reconstruction by biplane snakes. IEEE Transactions on Medical Imaging 21 (2002) 1188-1201

7. Byrne, J., Colominas, C., Hipwell, J., Cox, T., Noble, J., Penney, G., Hawkes, D.: An assessment of a technique for 2D-3D registration of cerebral intra-arterial angiography. British Journal of Radiology (2003) 